\title{
FROM FUNCTIONAL DYSPEPSIA TO CELIAC DISEASE
}

\section{CASE REPORT}

\author{
Lecturer. Dr. Ciocâlteu Adriana, \\ gastroenterologist, UMF Craiova, Medlife Sama Craiova Hyperclinic \\ Dr. Ionescu Mircea, \\ anesthesiology and intensive care consultant physician, Craiova County Emergency Clinical Hospital, \\ Medlife Sama Craiova Hyperclinic
}

\begin{abstract}
Stomach bloating is experienced by many young patients, which can lead to feelings of discomfort and pain. While there are instances when it would just be functional, there are cases when it would mean a more serious health problem. There has been a substantial increase in the prevalence of celiac disease over the last years and many patients remain undiagnosed. Diagnostic testing, including serology and biopsy, should be performed on a gluten-containing diet. The diagnosis and monitoring of celiac disease is complex and should be performed by a physician with special expertise.

The purpose of this paper is to describe the management of celiac disease in a young female student presenting symptoms suggestive of functional dyspepsia and to emphasize the idea that it is important to diagnose celiac disease in patients with a less clear clinical picture, because the disease may have negative health consequences in the future.
\end{abstract} disease

Key words: functional dyspepsia, celiac

\section{INTRODUCTION}

Functional dyspepsia is one of the most common digestive disorders in young people, and its prevalence is slightly increased in females. This is defined as the presence of dyspeptic symptoms without an organic cause. Sometimes, differential diagnosis in young patients with seemingly trivial symptoms can be a real challenge.

Over the last 50 years, there has been a substantial increase in the prevalence of celiac disease worldwide, yet many patients remain undiagnosed, thus stressing the need for new strategies for optimal patient detection [1]. The diagnosis of celiac disease is a complex one, based on a combination of clinical, serological and histopathological data, and the confirmation of the diagnosis, including serology and duodenal biopsy, should be made while under a gluten-containing diet.

Unlike functional disorders, regular monitoring of patients with celiac disease is important to be performed by a gastroenterologist or pediatrician experienced in the management of this pathology.

\section{CASE PRESENTATION}

We present the case of a 25-year-old patient from an urban environment, a medical student, who addressed the Gastroenterology Clinic for bloating and intermittent epigastralgia, symptoms not influenced by meals and that did not resolve after defecation or gas emissions. The patient indicates the onset of symptoms as being several years ago, during adolescence, insidiously, with progressive accentuation in the latest 3 months, especially during the exam session.

* Corresponding author: Ciocâlteu Adriana, UMF Craiova, Medlife Sama Craiova Hyperclinic, e-mail: adriana_ciocalteu@yahoo.com

Article received: 28.04 .2021 , accepted: 16.06 .2021 , published: 10.07 .2021

Cite: Ciocalteu A, Ionescu M. From functional dyspepsia to celiac disease - case report Journal of School and Univeristy Medicine. 2021;VIII(2):5-8 
The patient lives with her parents in an apartment in the city where she is a student, she does not smoke, she consumes coffee daily, and occasionally energy drinks and fast food products.

Both the personal pathological antecedents and the heredocolateral antecedents are insignificant.

As a background medication, she indicates the usage in the last two weeks of omeprazole $40 \mathrm{mg}$ daily, 30 minutes before breakfast and alverine / simeticone citrate three tablets daily between main meals, on the recommendation of the family doctor, without a considerable relief of symptoms.

At the clinical examination, the patient is in good general condition, normal weight, afebrile, with normally colored skin and mucous membranes, cardio-respiratory balanced, with a supple abdomen, mobile with respiratory movements and painless during palpation, liver and spleen clinically within normal limits, physiological urination, intestinal transit present (one stool / day of normal consistency).

Based on anamnestic and clinical data, we initially focused on functional dyspepsia. Characteristics and duration of symptoms of at least 6 months, constant in the latest 3 months in a young patient, in the absence of clinical elements suggestive of a possible organic lesion, as well as the patient's mention of the influence of stress during exams period (Rome criteria III fulfilled) initially imposed a hygienic-dietary regime and the continuation of a therapeutic cure, without other additional investigations.

The patient returned to the clinic after another two weeks in which she underwent cytoprotective treatment (bismuth oxide 120mgx 4 / day), trimebutine $100 \mathrm{mg} \times 3 /$ day and probiotics, without any improvement. Usual laboratory tests, coproparasitic examination, Giardia antigen in faeces, urine summary examination and urine culture were within normal limits. Also, the electrocardiogram and abdominal ultrasound did not show pathological changes.

Upper digestive endoscopy was performed under sedation with propofol, which showed a normal-looking esophagus and stomach (rapid urease test for Helicobacter Pylori negative at 15 minutes), and in the duodenum - nodular appearance of the mucosa, with crenellated folds and flattening of the villi (Image 1, Image 2). Multiple biopsies were taken, the endoscopic appearance being suggestive for gluten enteropathy. The pathological examination described the duodenal mucosa with atrophy of the villi and rich inflammatory infiltrate with lymphocytes extending to the covering epithelium.

The patient's Ig A anti-tissue transglutaminase antibodies showed elevated values $(200 \mathrm{U} / \mathrm{ml})$.

Thus, based on the endoscopic aspect, the histopathological examination and the presence of specific antibodies, the diagnosis of celiac disease was established.

The patient was recommended a gluten-free diet all her life and was encouraged to seek nutritional counseling and enroll in celiac disease communities or groups.

Endoscopic reassessment after one year, with the normalization of the duodenal mucosa (Image 3) and the undetectable titer of Ig A anti-tissue transglutaminase antibodies confirmed the young patient's compliance with the diet.

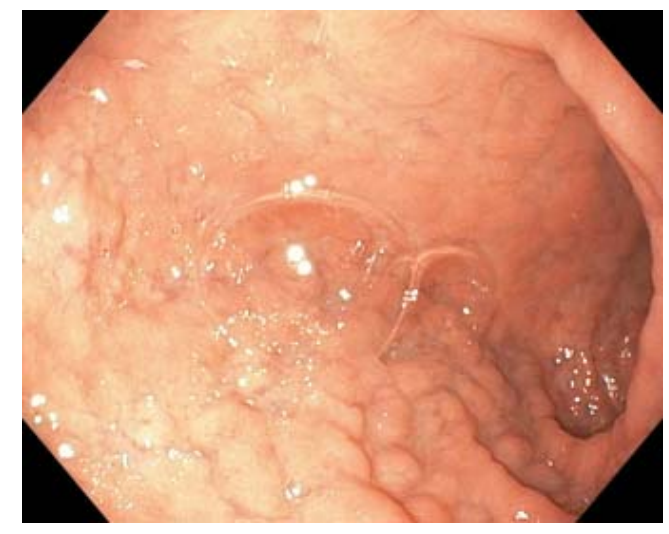

Image 1. Granular-looking duodenal bulb mucosa

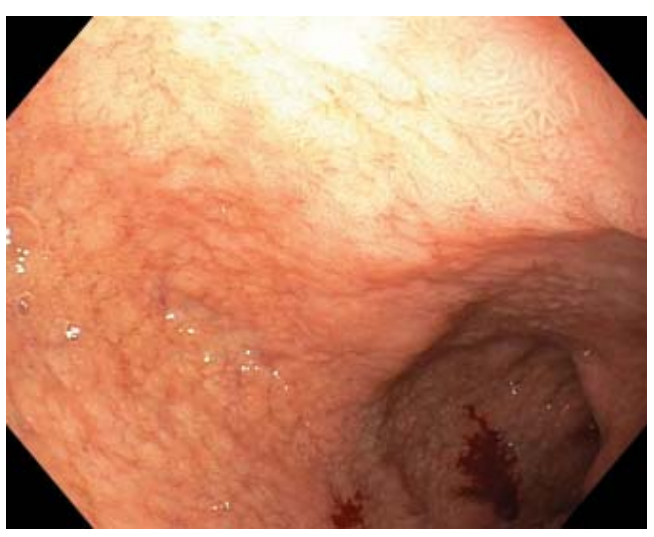

Image 2. Duodenal mucosa with flattened villi and lacy, crenellated appearance of the Kerckring folds 


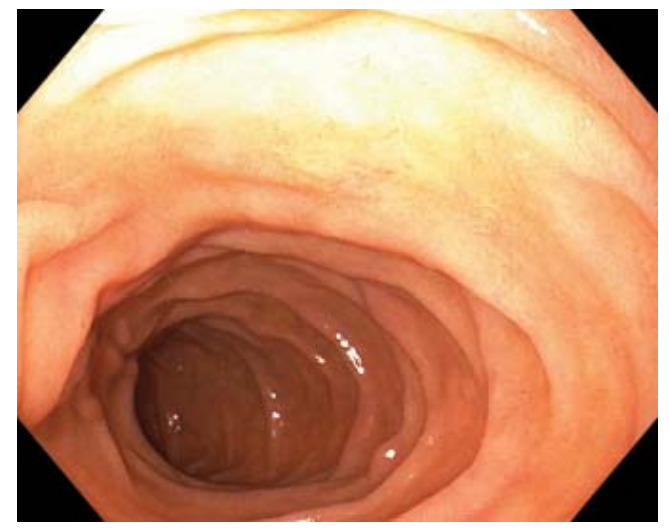

Image 3. Endoscopic image in the same patient after one year of gluten-free diet. Duodenal mucosa with villi and folds of normal appearance

\section{DISCUSSIONS}

According to the Rome III diagnostic criteria for functional dyspepsia, the symptoms must be constantly present in the latest 3 months, with the onset of symptoms at least 6 months before diagnosis. The Rome III consensus included in this category three subtypes, with possibly different pathophysiology and etiology: postprandial discomfort syndrome (symptoms induced by food ingestion), epigastric pain syndrome (symptoms not associated with food ingestion) and mixed syndrome [2].

Functional dyspepsia is not the result of a basic structural abnormality, but rather the consequence of multiple pathophysiological mechanisms, such as abnormal gastric motility, gastric and duodenal hypersensitivity to acid and / or post-Helicobacter pylori infection [3], psychosocial factors or a genetic predisposition to an exaggerated gastrointestinal response to the action of normal intensity psychic and physical factors.

The evolution of people with functional dyspepsia is favorable, with periods of calm and exacerbations, but without influencing the life prognosis.

While in dyspeptic patients over 45 years of age or in those with alarm signals (such as anemia, hereditary history of cancer) additional investigations are required to exclude an organic pathology, in young patients upper digestive endoscopy has been reserved for those without symptom relief after 6-8 weeks of symptomatic therapy (most commonly, proton pump inhibitors and prokinetics).
Intolerance to some foods remains controversial in functional dyspepsia and is most likely due to visceral hypersensitivity.

On the other hand, gluten consumption (present in foods based on wheat, rye and barley) has been linked to a number of clinical disorders, collectively referred to as gluten-related disorders, which have gradually become a relevant phenomenon in terms of epidemiological view [4].

Celiac disease (gluten enteropathy, celiac sprue) is a chronic multi-organic disease in which there is an immune-mediated reaction to gluten, affecting the small intestine of patients with a certain genetic predisposition. The prevalence of the disease is estimated at $0.5-1 \%$ of the general population [5], and the incidence is increasing in recent years [6].

Over $70 \%$ of patients are diagnosed after the age of 20 [1], some of them probably had the disease undetected since childhood, while others developed the disease in adulthood, on a predisposing background. Thus, loss of gluten tolerance can occur at any time in life as a consequence of other triggers (eg gastrointestinal infections, medications, $\alpha$-interferon, surgery).

This condition can have many forms, including gastrointestinal symptoms (eg, diarrhea, steatorrhea, weight loss, bloating, flatulence, abdominal pain) and / or other changes (abnormal liver function tests, iron deficiency or megaloblastic anemia, arthralgia), dermatological damage (herpetiform dermatitis, non-Hodgkin's lymphoma) [7].

In children, celiac disease is frequently manifested by growth retardation, diarrhea, muscle atrophy, whimsical appetite and abdominal distension. Many of these children also show signs of emotional distress, mood swings and lethargy, and others may have constipation and abdominal pain. The oligosymptomatic or asymptomatic forms of the disease are found especially in adulthood.

Celiac disease is usually detected by serological testing of specific antibodies, and the diagnosis is confirmed by biopsies of the duodenal mucosa. Both serology and biopsy should be performed while under a gluten-containing diet. There is still no test with perfect specificity or sensitivity. Currently, tissue anti-transglutaminase antibodies are the most sensitive serological test, preferred at any age for the detection 
of celiac disease, along with Ig A testing (to rule out a pre-existing Ig A deficiency), but also with a role in subsequent monitoring of compliance with gluten-free diet. In addition, as Helicobacter Pylori or Giardia infection can produce similar inflammatory histopathological changes and even false positive serological tests, they should be excluded.

The classic treatment for celiac disease consists primarily of a gluten-free diet for life, which can be a challenge especially for those diagnosed in adulthood. Thus, sustained patient education, motivation and monitoring are needed [8].

Adherence to a proper gluten-free diet is very important in these patients, as it leads not only to improved symptoms and healing of the duodenal mucosa, but also to reduced risk of malignancy (eg lymphomas), cardiovascular disease, infertility and miscarriages [9].

Wheat allergy can be considered for differential diagnosis, but it is not necessary for these patients to restrict dietary rye, barley or oats as in those with celiac disease. As a different entity from celiac disease, non-celiac gluten sensitivity is a controversial topic, in which patients do not have gluten allergy or changes in the intestinal mucosa, but may benefit to varying degrees from symptoms improvement under the restrictive diet [10].

In recent years, the gluten-free diet has gained ground, including among patients with irritable bowel syndrome, with an improvement in their symptoms following the option of restrictive diet. It is important to remember, however, that the initiation of such a diet without the advice of a specialist can mask or further delay the correct diagnosis and management of pathologies with organic substrate, such as celiac disease.

\section{CONCLUSIONS}

The present case illustrates a currently underdiagnosed pathology and the importance of upper digestive endoscopy as a means of guiding the diagnosis in the context of the persistence of a common symptomatology in young patients.

\section{REFERENCES}

1. Al-Toma A, Volta U, Auricchio R, Castillejo G, Sanders DS, Cellier C, Mulder CJ, Lundin KEA. European Society for the Study of Coeliac Disease (ESsCD) guideline for coeliac disease and other gluten-related disorders. United European Gastroenterol J. 2019 Jun;7(5):583-613. doi: 10.1177/2050640619844125. Epub 2019 Apr 13. PMID: 31210940; PMCID: PMC6545713.

2. Tack J, Talley NJ. Functional dyspepsia--symptoms, definitions and validity of the Rome III criteria. Nat Rev Gastroenterol Hepatol. 2013 Mar;10(3):134-41. doi: 10.1038/nrgastro.2013.14. Epub 2013 Feb 12. PMID: 23399526.

3. Giurcan R, Voiosu TA. Functional dyspepsia: a pragmatic approach. Rom J Intern Med. 2010;48(1):9-15. PMID: 21180236.

4. Kurppa K, Paavola A, Collin P, Sievänen H, Laurila K, Huhtala H, Saavalainen P, Mäki M, Kaukinen K. Benefits of a gluten-free diet for asymptomatic patients with serologic markers of celiac disease. Gastroenterology. 2014 Sep;147(3):610-617.e1. doi: 10.1053/j.gastro.2014.05.003. Epub 2014 May 13. PMID: 24837306.

5. Fasano A, Berti I, Gerarduzzi T, et al. Prevalence of celiac disease in at-risk and not-at-risk groups in the United States: a large multicenter study. Arch Intern Med. 2003;163(3):286- 292

6. Rubio-Tapia A, Kyle RA, Kaplan EL, et al. Increased prevalence and mortality in undiagnosed celiac disease. Gastroenterology.2009;137:88-93.

7. Hervonen K, Salmi TT, Ilus T, Paasikivi K, Vornanen M, Laurila K, Lindfors K, Viiri K, Saavalainen P, Collin P, Kaukinen K, Reunala T. Dermatitis Herpetiformis Refractory to Gluten-free Dietary Treatment. Acta Derm Venereol. 2016 Jan;96(1):82-6. doi: 10.2340/00015555-2184. PMID: 26084552.

8. Rubio-Tapia A, Hill ID, Kelly CP, Calderwood AH, Murray JA; American College of Gastroenterology. ACG clinical guidelines: diagnosis and management of celiac disease. Am J Gastroenterol. 2013 May;108(5):656-76; quiz 677. doi: 10.1038/ajg.2013.79. Epub 2013 Apr 23. PMID: 23609613; PMCID: PMC3706994.

9. Fasano A, Sapone A, Zevallos V, Schuppan D. Nonceliac gluten sensitivity. Gastroenterology. 2015;148(6):11951204. doi:10.1053/j.gastro.2014.12.049.

10. Pietzak M. Celiac disease, wheat allergy, and gluten sensitivity: when gluten free is not a fad. JPEN J Parenter Enteral Nutr. 2012 Jan;36(1 Suppl):68S-75S. doi: 10.1177/0148607111426276. PMID: 22237879. 